\title{
Decision-making at a T-junction by gradient-sensing microscopic agents
}

\author{
Tanvi Gandhi, Jinzi Mac Huang, Antoine Aubret, Yaocheng Li, Sophie Ramananarivo, \\ Massimo Vergassola $\odot,{ }^{\dagger}$ and Jérémie Palacci $\odot^{\star}$ \\ Department of Physics, University of California San Diego, La Jolla, California 92093, USA
}

(Received 1 April 2020; accepted 1 September 2020; published 14 October 2020)

\begin{abstract}
Active navigation relies on effectively extracting information from the surrounding environment, and often features the tracking of gradients of a relevant signal-such as the concentration of molecules. Microfluidic networks of closed pathways pose the challenge of determining the shortest exit pathway, which involves the proper local decision-making at each bifurcating junction. Here, we focus on the basic decision faced at a T-junction by a microscopic particle, which orients among possible paths via its sensing of a diffusible substance's concentration. We study experimentally the navigation of colloidal particles following concentration gradients by diffusiophoresis. We treat the situation as a mean first passage time (MFPT) problem that unveils the important role of a separatrix in the concentration field to determine the statistics of path taking. Further, we use numerical experiments to study different strategies, including biomimetic ones such as run and tumble or Markovian chemotactic migration. The discontinuity in the MFPT at the junction makes it remarkably difficult for microscopic agents to follow the shortest path, irrespective of adopted navigation strategy. In contrast, increasing the size of the sensing agents improves the efficiency of short-path taking by harvesting information on a larger scale. It inspires the development of a run-and-whirl dynamics that takes advantage of the mathematical properties of harmonic functions to emulate particles beyond their own size.
\end{abstract}

DOI: 10.1103/PhysRevFluids.5.104202

\section{INTRODUCTION}

The importance of oriented motion in food searching, survival, and mating is a potent evolutionary force that led to the tinkering of effective and diverse strategies of navigation in the natural realm [1]. Slugs can faithfully sense concentration gradients across their bodies to direct them towards sources of nutrients [2]. This strategy does not lend itself to smaller organisms since the local spatial gradient is overwhelmed by noise and diffusion of the chemical [3]. Instead, bacteria such as E. coli ought to trade space for time, moving much faster, estimating the local concentration gradients by time differences, and orienting by proper modulations of their tumbling rate [4]. The size of the particles plays a similar discriminant role in the navigation of nonliving particles. As a matter of fact, existing methods for the identification of the shortest path in a maze involve big

\footnotetext{
${ }^{*}$ Present address: Ladhyx, Ecole Polytechnique, Institut Polytechnique de Paris, Palaiseau, France.

'Present address: Laboratoire de Physique de l'Ecole Normale Supérieure, ENS, Université PSL, CNRS, Sorbonne Université, Université de Paris, F-75005 Paris, France.

palacci@ucsd.edu
}

Published by the American Physical Society under the terms of the Creative Commons Attribution 4.0 International license. Further distribution of this work must maintain attribution to the author(s) and the published article's title, journal citation, and DOI. 
particles and global knowledge harvested from nonlocal quantities, for instance, millimetric droplets by the Marangoni effect [5,6], amoeboid growth [7], the propagation of chemical waves [8], trains of droplets advected by a solvent flow [9], or the parallel exploration of all possible paths in a maze by pressure-driven flows [10]. Microfluidic networks are further used to confine bioagents [11] and chemotactic bacteria, showing the formation of bacterial clusters where bacteria secrete their own chemoattractant [12] or the ability of bacteria to search out each other and dynamically confine themselves [13]. Branching maze geometries have been used in ecology to study the orientation of penguins or birds $[14,15]$ and the routing of plant roots [16] in response to volatile chemical compounds. In microfluidic settings, they offer controlled environments and chemical gradients to study the navigation of $C$. elegans worms [17] or reveal phenotypic heterogeneity in chemotactic sensitivity of bacteria [18].

Our distinct goal here is to address the navigation of microfluidic networks by small particles that orient and displace by active sensing, which prevents them from being simply carried by the flow. We consider T-junctions, with bidirectional concentration gradients, which can lead to to one or the other branch, differing by the amplitude of the gradient. This problem of navigation is the path-taking agents entering the junction to follow the gradient along one branch or the other, in contrast to the ecology examples discussed above. In this situation, the microscopic size of the particles poses new challenges in sensing, similar to those mentioned above for bacteria as compared to bigger eukaryotic cells. In addition to those fundamental reasons, the problem is also motivated by applications to microrobotics, where reduced sizes are favored in order to reduce invasiveness and local searches are needed for targeted delivery [19,20]. For particles with a sensing mechanism leading to a mean migration velocity $\mathbf{v} \propto \nabla f(s)$, where $f$ is a reasonably smooth function of a chemical $s$ diffusing throughout the network, a global constraint of vanishing circulation, $\oint \mathbf{v} \cdot d \mathbf{l}=$ 0 , holds on every closed contour. A priori, this would imply a higher velocity along the shortest available path in the maze, similarly to hydrodynamic flows [9]. However, hydrodynamic flows are deliberately absent in our setup, as mentioned above. Instead, particles must find their way by sensing local gradients of the chemical $s$ and by using them to make their decisions at the successive junctions of the maze.

In this work, we study the statistics of path taking at T-junctions, where microscopic agents interact with steady concentration gradients imposed via flowing solutions connected to the inlet and outlet of a microfluidic network. In such configurations, gradient-sensing particles travel to the exit while discounting dead ends that exhibit uniform concentration - a feature recently highlighted for chemotactic cells "seeing around the corner" [21]. As a result, particles navigate along pathways that connect the entry to the exit of a microfluidic network. We determine how the series of successive local decisions at the T-junctions affect global navigation and the capacity to effectively identify optimal paths. We first investigate the phenomenon with a model system of colloidal beads directed along a gradient of electrolyte by diffusiophoresis, an interfacial phenomenon [22-26] leading to the migration of particles with velocity $\mathbf{v}=D_{D P} \nabla \ln c$. Here, $c(\mathbf{x}, t)$ is the total electrolyte concentration with temporal $(t)$ and spatial $(\mathbf{x})$ dependence, and $D_{D P}$ is the diffusiophoretic mobility of the particle.

We study the navigation of those particles in a microfluidic network and the statistics of path selection, which we compare with theoretical and numerical models. Specifically, we treat the navigation as a mean first passage time (MFPT) problem, which allows us to unveil the existence of discontinuities in the MFPT that determines the decision-making at junctions. We further investigate different navigation strategies to improve the agents' performance to follow the optimal (shortest) path, including run-and-tumble and the Markovian chemotactic dynamics. We show that they all exhibit relatively poor performance, which we trace back to the size of the agents by showing how the performance improves with their dimensions. Finally, we illustrate this effect with a toy model of "run-and-whirl" of particles performing straight runs combined with random left- or right-handed turns circles, emulating the navigation of particles of larger size. 

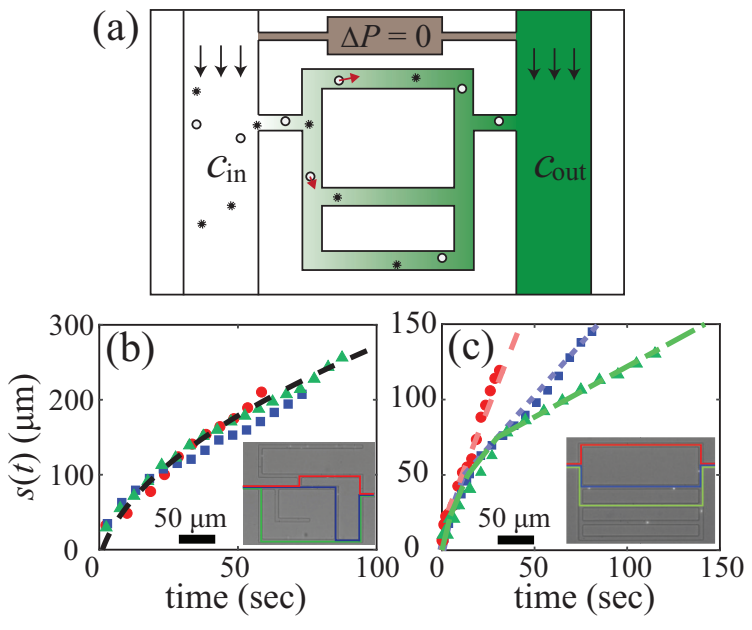

FIG. 1. (a) Sketch of the experimental setup. Continuous lateral flows set the boundary conditions and allow the development of a steady concentration map in the microfluidic network. Colloids (white particles) added to the lateral flow enter the network by diffusiophoresis, migrating towards the high concentration of salt. Engineered "hairy" colloids do not exhibit diffusiophoretic migration and allow one to ensure the absence of transversal flow as controlled by a pressure controller. The color map represents the steady concentration map of the Laplace equation $\Delta c=0$. (b),(c) Curvilinear displacement of colloidal particles by diffusiophoresis. (b) In the absence of background salt, the time dependence of the curvilinear position is independent of the path (color for the paths is defined in the inset). The experimental results are in good agreement with the logarithmic sensing, $\mathbf{v}=D_{D P} \nabla \ln c$. (c) With the presence of background salt $\bar{c}$, the time dependence of the curvilinear position depends on the path (the color for the paths is defined in the inset). The speed on each segment is constant, consistent with the linear sensing, $\mathbf{v}=\frac{D_{D P}}{\bar{c}} \nabla c$.

\section{RESULTS AND DISCUSSION}

We first study the navigation of colloidal particles introduced into a microfluidic network made of polydimethylsiloxane (PDMS) (see the Appendices). We consider the network constituted by channels of constant height and width, and varying lengths [Figs. 1(a) and 2(a)]. Large lateral channels connect to the entrance (source) or exit (sink) of the solute, allowing the solute to diffuse through the network and establish a steady concentration field that satisfies the Laplace equation $\Delta c=0$ [Fig. 1(b)].

Special attention was taken to suppress solvent flows inside the network: the reduced height of the microfluidic channels, $h \sim 5 \mu \mathrm{m}$, provides a large hydrodynamic resistance $\propto 1 / h^{3}$, and a precision pressure controller (Fluigent), which imposes boundary flows and compensates for the pressure imbalance in real time. Furthermore, solvent flows in the channels are monitored by control microparticles engineered to exhibit vanishing diffusiophoretic mobility (see the Appendices) [27]. All experiments are performed in buffer (tetramethylammonium hydroxide salt, TMAH, $c_{b}=0.6 \mathrm{mM}$ ) with $\mathrm{pH} \sim 9$, which sets the surface chemistry of the particles.

Particles of interest are $1 \mu \mathrm{m}$ fluorescently labeled beads that are introduced via the boundary channels [Fig. 1(a), white particles]. They enter the microfluidic network as diffusiophoresis drives them towards the high concentration of salt. The control particles with a grafted polymer brush [black particles in Fig. 1(a)] only exhibit Brownian motion, indicative of the absence of solvent or osmotic flows (see movie S1 in the Supplemental Material [41]). We observe the motion of the particles by conventional fluorescent microscopy to extract the curvilinear position $s(t)$ [28]. In each channel, the steady concentration of $\mathrm{LiCl}$ is a solution of the Laplace equation, $\nabla^{2} c=0$, leading to $c(s)=c_{\min }+s \Delta c / L_{i}$, where $L_{i}$ is the curvilinear length of each path, $c_{\min }$ and $c_{\max }$ are, 

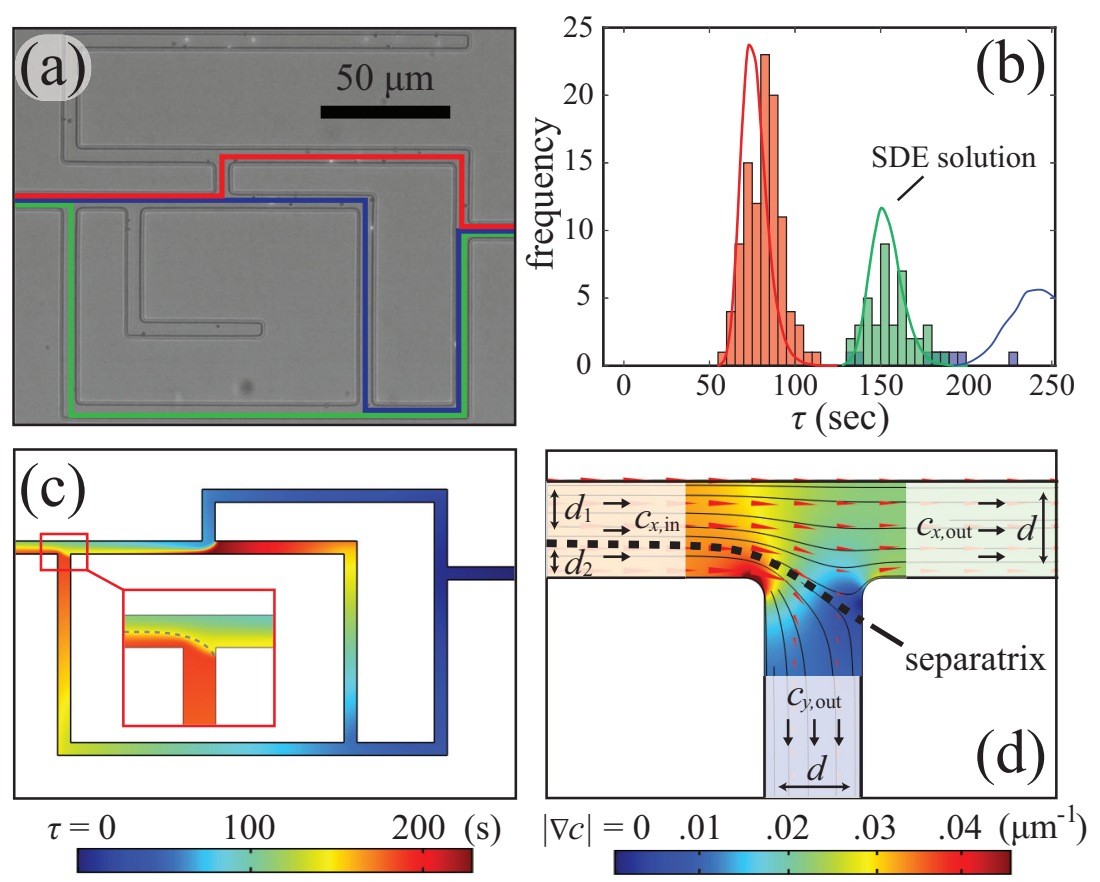

FIG. 2. (a) Microfluidic network. Three different paths are coded with different colors, with red the shortest and blue the longest. (b) Histogram of exit time for particles traveling through the microfluidic network, where each path has the same color scheme as in (a). The histogram agrees well with the stochastic modeling (solid lines). (c) Numerical solution of the mean first passage time (MFPT) in the geometry of (a). The discontinuity in the MFPT is highlighted in the inset. (d) Discontinuity of MFPT is prescribed by a separatrix in the concentration field, whose position is set by the geometry and the relative gradients in paths exiting the T-junction.

respectively, the entrance and exit concentration, and $\Delta c=c_{\max }-c_{\min }$. The magnitude of phoretic velocity is then $\left|\mathbf{v}_{D P}\right|=D_{D P}|\nabla \ln c(s)|=\Delta c /\left[L_{i} c_{\text {min }}+s \Delta c\right]$. So there are two limits for the profile of $\mathbf{v}_{D P}$ : when $\Delta c \gg c_{\min },\left|\mathbf{v}_{D P}\right| \approx 1 / s$ is independent of the path length and concentration; and when $\Delta c \ll c_{\min },\left|\mathbf{v}_{D P}\right| \approx \Delta c /\left(c_{\min } L_{i}\right)$ is independent of $s$.

We first consider the situation $\Delta c \gg c_{\text {min }}$ by imposing a gradient of $\mathrm{LiCl}$ with $c_{\min }=0 \mathrm{mM}$ and $c_{\max }=6 \mathrm{mM}$. We observe $s(t)$ as shown in Fig. 1(b), exhibiting a higher particle velocity near the entrance than further down the path. The diffusiophoretic mobility is determined by integrating $d s / d t=\left|\mathbf{v}_{D P}\right|=D_{D P} / s$ and fitting to the experimental trajectories, which yields $D_{D P} \sim 380 \mu \mathrm{m}^{2} / \mathrm{s}$ that is comparable to previously reported mobilities in $\mathrm{LiCl}$ gradients for particles of comparable surface chemistry [24]. Remarkably, the particle velocity does not depend on the choice of the path [Fig. 1(b)] and does not involve the magnitude of the concentration gradient $\Delta c / L_{i}$, in contrast with other phenomena such as salt trapping [24] and persistent particle removal [29]. The logarithm sensing suggests that diffusiophoretic motion remains effective down to very small salt concentrations and gradients, provided that $\nabla c / c$ remains finite-a feature previously reported in the experiments of "osmotic shocks" where particles migrate in vanishing concentration gradients and vanishing concentration [30]. The limit of this counterintuitive result-in the context of an ultradilute salt where the intra-ion distance compares with the particle size-remains to be investigated.

The path-independence property is lost when adding a background concentration of salt, $\bar{c}=5$ $\mathrm{mM}$, obtained using $c_{\min }=4 \mathrm{mM}$ and $c_{\max }=6 \mathrm{mM}$. The particle velocity becomes $\left|\mathbf{v}_{D P}\right|=$ $\left(D_{D P} / \bar{c}\right)|\nabla c|$, where $\bar{c}=5 \mathrm{mM}$ is the average $\mathrm{LiCl}$ salt concentration and $\nabla c$ is a constant in 
each segment of the network, dependent on the geometry of the network and $\Delta c$. The curvilinear displacement of the particles is shown in Fig. 1(c), and the piecewise linear displacement confirms the constant velocity in each segment of the path and is higher in shorter channels. Noticeably, a reduced number of particles enters the microfluidic network in this situation, even using smaller particles, showing that it is not a result of spatial hindrance. Furthermore, the diffusiophoretic mobility measured for the particles navigating the microfluidic network, $D_{D P}^{*} \geqslant 1000 \mu \mathrm{m}^{2} / \mathrm{s}$, is significantly larger than for the case of no background salt [Figs. 1(b) and 1(c)]. We attribute this observation to a sorting mechanism analogous to the diffusiophoretic capture of particles in dead-end pores recently discussed by Battat et al. [25]. In brief, only particles that exhibit a sufficient transverse drift in the concentration gradient at the entrance, $\left|\mathbf{v}_{D P}\right|=D_{D P}^{*} /\left.\bar{c} \nabla c\right|_{s=0}$, can escape flow streamlines of the boundary channels and enter the microfluidic network. It requires that entering particles have higher diffusiophoretic mobilities $D_{D P}^{*}$ with salt background rather than without, for which the entrance velocity is $\left|\mathbf{v}_{D P}\right|=\left.D_{D P} \nabla \ln c\right|_{s=0}$ [Figs. 1(b) and 1(c)]. It results in an effective sorting, where only particles with high diffusiophoretic mobilities enter the network with the presence of background salt. The origin of this difference in diffusiophoretic mobility, which cannot be simply accounted for by differences in size [31], is likely related to differences in surface chemistry and $\zeta$ potential in the colloidal suspension. A detailed analysis of this effect is, however, beyond the scope of the present work.

Next, we investigate the navigation of particles in microfluidic networks, where the possible paths are color coded by length [Fig. 2(a)]. The histogram of the exit times for particles choosing the different paths is shown in Fig. 2(b), where the shortest (red) path exhibits the shortest exit time and a much higher probability. It indicates a selectivity in the path taking, which we now aim to explain through stochastic modeling. The particle motion has two contributions: (i) a diffusiophoretic drift, $\mathbf{v}_{D P}=D_{D P} f(c) \nabla c$, and (ii) a random component of Brownian motion, $\sqrt{2 D} W_{t}$, where the diffusion coefficient, $D \approx 0.4 \mu \mathrm{m}^{2} / \mathrm{s}$ for $1 \mu \mathrm{m}$ particles, is estimated from the Stokes-Einstein relation [32]. The resulting equation for the position of the particle reads $d \mathbf{X}_{t}=D_{D P} f(c) \nabla c d t+\sqrt{2 D} d W_{t}$. Without background salt, $f(c)=1 / c$, and the integration of this Itô stochastic differential equation (SDE) (see the Appendices) yields the distribution shown in Fig. 2(b), which captures the experimental statistics. The equilibrium statistics is determined analytically via the associated steady-state Fokker-Planck equation $\nabla \cdot\left[-D_{D P} f(c) \rho \nabla c\right]+D \Delta \rho=0$, which can be solved exactly for the particle density $\rho(\mathbf{x}, t)$. The associated probability current is given by $\mathbf{j}=\gamma \nabla c$, where $\gamma$ is a constant determined by the boundary conditions (see the Supplemental Material [41]). As a result, the number of particles choosing each path is proportional to the concentration gradient, which explains why more particles choose the shortest path [Figs. 2(a) and 2(b)].

Defining the exit time $t_{e}(\mathbf{x})$ of a particle started at position $\mathbf{x}$ in the network, its expectation is the mean first passage time (MFPT) $\tau(\mathbf{x})=\mathbb{E} t_{e}$, which satisfies $D_{D P} f(c) \nabla c \cdot \nabla \tau+D \Delta \tau=-1$ with $\partial_{n} \tau=0$ at the maze wall and $\tau=0$ at the entrance and exit [33]. The MFPT is solved numerically (Fig. 2(c); see Supplemental Material [41]) and exhibits a discontinuity at each junction, reflecting the different paths taken by the particles [inset of Fig. 2(c)]. To explain this discontinuity, we plot the color map of the magnitude of salt concentration gradient, with the direction of $\nabla c$ labeled by red triangles [Fig. 2(d)]. It shows a separatrix between the two paths, along which $\nabla c$ is tangential, and creates a discontinuity of the value for $\tau$. In the absence of Brownian noise, particles do not exchange between each side of the separatrix, leading to a sharp discontinuity of $\tau$ at the separatrix and the formation of a shock. In this case, the conservation of mass leads to $d_{1}\left|c_{x, \text { in }}\right|=d\left|c_{x, \text { out }}\right|$ and $d_{2}\left|c_{x, \text { in }}\right|=d\left|c_{y, \text { out }}\right|$, using the notations from Fig. 2(d) and where $c_{x}$ and $c_{y}$ are the gradient of the concentration $c$ along $x$ and $y$, respectively. For a uniform incoming density, the ratio of the particle number choosing each branch is then $N_{1} / N_{2}=d_{1} / d_{2}=\left|c_{x, \text { out }}\right| /\left|c_{y \text {,out }}\right|$, reaching the same conclusion as the Fokker-Planck approach: the particle number at each path is proportional to the magnitude of salt gradient there. The discontinuity of the MFPT clearly identifies the origin of path taking at the junction. Since it originates from the separatrix in the steady concentration field, we do not expect the statistics of path taking to depend on the amplitude of the diffusiophoretic mobility, provided it is nonzero (see the Supplemental Material [41]), though the exit time would. As a result, 


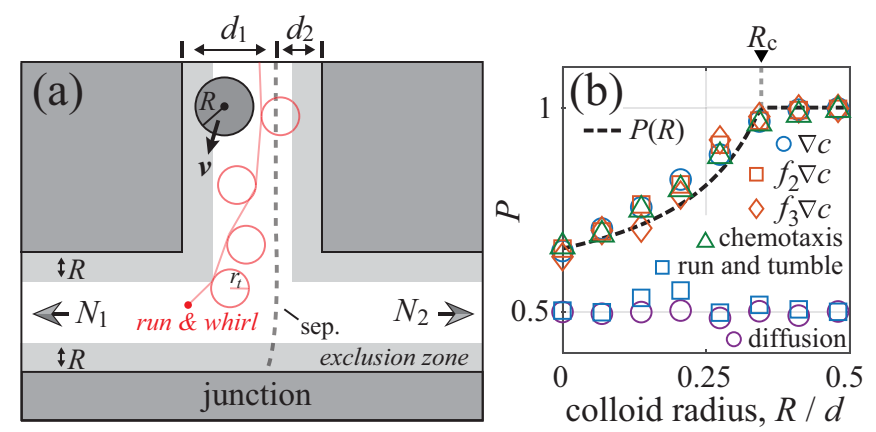

FIG. 3. (a) The efficiency of the path taking for particles with radius $R$ is improved due to the exclusion zone (shaded area) near the wall. A "run-and-whirl" navigation scheme (red trajectories) based on the mathematical properties of the analytic is proposed, which allows point particles to emulate the performance of particles with larger radii. (b) Path selectivity $P$ for different navigation strategies (different symbols). $f_{2}=1 / c$ represents the phoretic strategy and $f_{3}=\mathbf{1}_{c>\bar{c}}$ represents a strategy that has a nonsmooth dependence on $c$. Chemotactic migration is obtained by evolving a three-state system, run-and-tumble strategy and pure diffusion give roughly $50 \%$ selectivity at the junction. All strategies but the run and tumble and pure diffusion compare similarly and agree with our analytic prediction for particles of finite size (dashed line). The selectivity for point particles at the considered gradient is $P(0)=d_{1} / d=0.65$, with the critical particle size $R_{c}=d_{2}=0.35 d$, beyond which the selectivity is 1 .

the statistics of path taking should remain unchanged in the general case where the diffusiophoretic mobility depends on the concentration, $D_{D P}(c)$, for example, when accounting for the variations of diffusiophoretic mobility of particles of radius $a$ with thin and finite Debye layer of thickness $\kappa^{-1}$ [31,34] (see the Supplemental Material [41]).

So far, we have considered the path taking of small particles, whose spatial extension is small compared to the dimensions (width or height) of the microfluidic network. We now turn to the effect of particles size on the navigation scheme. Here, we simply consider the walls as impenetrable boundaries setting the geometry of the network. The effects of the walls on continuum fluid mechanics (hydrodynamic interactions) and solute transport can be neglected as a result of their leading-order scaling $\propto(a / h)^{3}$, where $a$ is the particle radius and $h$ is the distance from the particle center to the (nearest) wall (see the Supplemental Material [41]) [35,36]. The phoretic velocity of a particle with finite radius $R$ is obtained by averaging the slip velocity along the particle surface, $\mathbf{v}(x, y)=\left\langle D_{D P} f(c) \nabla c\right\rangle$ [22,36]. In the presence of background salt, $f=1 / \bar{c}$ is a constant, the velocity of the particle of finite size is given by the value of the gradient at the center, $\mathbf{v}=\left(D_{D P} / \bar{c}\right) \nabla c$, as reported in [22,36], which can be formally understood from the components of $\nabla c$ being harmonic functions and satisfying the mean value principle (MVP) - the spherical three-dimensional (3D) or the circular (2D) average of a harmonic function is its value at the center of the sphere or the circle. We extend this result to any smooth function $f(c)$, showing that $f(c) \nabla c$ almost satisfies MVP for harmonic functions as a result of the harmonic properties of the concentration field $c$. In that case, the correction is controlled by the aspect ratio of the network (see the Supplemental Material [41]). It follows that we can treat a particle of finite size, in the limit of thin Debye layers, the same way as a point particle positioned at the particle's center, as previously discussed in $[22,36]$. However, the nonoverlap condition of the particles with the walls creates an exclusion zone, as shown in Fig. 3(a). Assuming the particle enters the junction with a uniform density and the left exit has higher salt gradient, so that $d_{1}>d_{2}$, the probability of the particle exiting through the left side, or the selectivity, is $P(R)=\lim _{N \rightarrow \infty} N_{1} / N=\left(d_{1}-R\right) /(d-2 R)$, where $N=N_{1}+N_{2}$ is the total number of particles entered and $d=d_{1}+d_{2}$ is the channel width. The piecewise continuous $P(R)$ is plotted in Fig. 3(b) and presents a critical particle radius $R_{c}=d_{2}$, above which particles show perfect selectivity at the junction: $P(R)=1$, for $R>R_{c}$. 
We test this prediction numerically for particles of different sizes and different navigation strategies, arriving at a junction. We assume that the presence of the particle does not modify the concentration field obtained in the absence of particles, a reasonable assumption for dilute suspension of particles, whose thickness is small compared to the height of the channel, as in the experiment. Particles with velocity $\mathbf{v} \sim f \nabla c$ that has $f_{1}=1$ or $f_{2}=1 / c$ follow our prediction for $P(R)$ with an improved selectivity arising only as the particle size increases [Fig. 3(b)]. To test the robustness of this result, we consider functions $f$ that lack the regularity or depend on the history: (i) A piecewise continuous indicator function $f_{3}=\theta(c-\bar{c})$ that is unity when $c$ is greater than its mean on the boundary and vanishes otherwise; (ii) temporal chemotaxis implemented via run-and-tumble dynamics; (iii) spatial chemotaxis achieved from a three-state system that presents a hysteretic behavior [37] (see the Appendices). Remarkably, most navigation strategies present a selectivity in line with the simple geometric argument set by the concentration field [Fig. 3(b)]. Run-and-tumble navigation, however, performs poorly and compares with random diffusion, over a broad range of tumbling rates (see the Supplemental Material [41]). Though run and tumble is an efficient chemotactic strategy along smooth gradients, this result unveils the inadequacy of temporal chemotaxis to capture the spatial discontinuity set by the separatrix. Three remarks are in order. The first one is that the discontinuity in the direction of migration at the separatrix makes run and tumble different from the migration along $\nabla c$ [see Fig. 3(b)]. The second one is that this phenomenon is not observed in situations such as in Ref. [18], where the transverse component of the gradient in the junction is unidirectional and therefore no separatrix is present. Finally, we only considered the phoretic velocity component that has the same direction as $\nabla c$, while other considerations-such as the delays in the polarization of the active sites that can lead to displacements transverse to the concentration gradient - could lead to a tensorial mobility have not been studied in the present work.

The results presented in Fig. 3 show that bigger particles achieve higher selectivity, emphasizing the importance of averaging for adequate decision-making. Inspired by this result, we investigate whether a small particle can emulate a particle of larger size by an adequate navigation strategy. In line with the navigation strategies considered above, we assume that particles measure local concentration $c$ and gradient $\nabla c$ and have memory, so that $c$ and $\nabla c$ along the path of the particle are known. We are aiming to infer the distribution of $c$ at a junction through its value along a curve (trajectory of the particle), a problem formally known as the search for the analytic continuation of $c$, i.e., determine the value of a harmonic function in a domain $\Omega$ given its value in a subdomain $\Gamma \subset \Omega$. In general, this is numerically ill conditioned and one cannot estimate the value of the concentration everywhere by knowing the value of the concentration and gradient along a line. The concentration $c$ can, however, be determined through boundary integrals if the trajectory forms a closed loop. It inspires us in the design of "run and whirl," where particles complete straight runs of random lengths before making a random left- or right-handed turn and completing a full circle with radius $r_{t}$ [see Fig. 3(a) and Appendices]. Upon completion of the loop, the values of $c$ and $\nabla c$ are known along the circle, making it possible to determine $\nabla c$ at the center of the circle using Poisson's formula, and circumventing the above indetermination. Conveniently, this value is directly determined by averaging $\nabla c$ along the circular path as a result of the MVP. The particle then runs along the new direction set by this averaged $\nabla c$; the overall effect of this scheme is for a point particle to emulate a gradient-sensing particle with radius $r_{t}$.

The efficiency of this scheme depends on the tumbling rate $\beta$ and the turning radius $r_{t}$. At a moderate rate $\beta=1 \mathrm{~s}^{-1}$ and turning radius $r_{t}<0.1 d$, the point particle has enhanced selectivity at the junction as its navigation scheme effectively emulates that of a bigger particle with size $R=r_{t}$ (see the Supplemental Material [41]). We further test the robustness of the navigation by adding angular diffusion $D_{r}$ to the turning stage, such that particles are turning with a mean angular velocity plus noise. As a result, the agent follows loops rather than perfect circles (see typical trajectories in the Supplemental Material [41]). The results gathered in Table I show a range of enhancement of the selectivity compared to the efficiency of a point particle and that the selectivity for a particle with turning radius $r_{t}$ tracks the selectivity $P\left(r_{t}\right)$ of a particle of radius $r_{t}$, as discussed above and presented in Fig. 3(b). However, the effects of collisions with the walls dominate when the turning 
TABLE I. Comparison of path taking between particles of finite size and of run-and-whirl navigation. The first line is the efficiency $P\left(r_{t}\right)$ for a particle of radius $r_{t}$ [see main text; dashed line in Fig. 3(b)]. The remaining lines give the efficiency of the run-and-whirl strategy of point particles for varying whirling rate $\beta$, and angular diffusion of the particles $D_{r}$.

\begin{tabular}{lcccr}
\hline \hline Model & & & & \\
\hline$P\left(r_{t}\right)$ & 0 & 0.04 & 0.08 & 0.12 \\
$\beta=1 \mathrm{~s}^{-1}, D_{r}=0 \mathrm{~s}^{-1}$ & 0.66 & 0.67 & 0.69 & 0.70 \\
$\beta=1 \mathrm{~s}^{-1}, D_{r}=0.2 \mathrm{~s}^{-1}$ & 0.65 & 0.67 & 0.69 & 0.65 \\
\hline \hline
\end{tabular}

radius or the whirling rate further increases and limits the effective average of the scheme (see the Supplemental Material [41]). Remarkably, the effect is robust to the addition of noise (Table I), a result of the harmonic properties of the concentration, allowing an adequate estimate of the concentration on any closed-loop trajectories. Though the range of application and improvement remains limited, the run-and-whirl navigation is primarily proposed as a toy model for its conceptual value, showing how mathematical properties of harmonic functions can be leveraged to allow particles to sense beyond their own size. For such a strategy to be effective, the persistence of the motion needs to be sufficiently large. As a result, the implementation of such strategy for agents smaller than 1-2 $\mu \mathrm{m}$ appears problematic and will likely be dominated by angular noise. Recent experimental realizations above this scale have demonstrated that memoryless "run and whirl" at the microscale is within reach, using, for example, micrometric nanorods with curvature [38] or self-propelled particles with anisotropic drag [39] to curve trajectories. Those systems were, however, missing the integration of signal over their strategies to adequately implement our proposed navigation. Run and whirl highlights the importance of averaging in space to gather information and capture the separatrix, resulting in better selectivity at the junction. A conceptual asset of the navigation is its use of the harmonic properties of the concentration field, which allows the strategy to remain valid in the presence of angular noise, when particles form closed-loop trajectories rather than circles, and providing a pathway to enhanced navigation efficiency.

\section{CONCLUSION}

We demonstrated that synthetic colloids can navigate through microfluidic networks by gradientsensing diffusiophoresis. We show that the statistics of path taking and exit time of small particles are accurately captured by a mean-field approach to the corresponding mean first passage time problem, borrowed from stochastic physics. It highlights that the separatrix in the concentration field leads to a sharp jump in the mean first passage time, which is the key component that sets the effectiveness of the navigation scheme. Our study was further extended to particles of larger size. Using numerical experiments, we demonstrated that a broad class of navigation schemes along $\nabla c$ behaves similarly, since they share a common separatrix set by the concentration field and independent of the navigation strategy. The discontinuous nature at the separatrix illustrates the difficulty of the decision process at a junction for microscopic agents that have to rely on gradient sensing and are not carried by any directed flow. Finally, we proposed a scheme that demonstrates the possibility of improving the navigation scheme of small agents at a junction. Although the experimental implementation of the proposed method remains elusive and is limited to navigations with sufficient directional persistence, it shows that one can harness the harmonic properties of the concentration field to improve navigation even in the presence of noise. 


\section{ACKNOWLEDGMENTS}

We thank S. Sacanna and M. Youssef for the synthesis of pluronic particles. The experiments, experimental data acquisition, and analysis were supported by the National Science Foundation under Grant No. DMR-1554724. The stochastic modeling and numerical simulations were supported by the U.S. Department of Energy (DOE), Office of Science, Basic Energy Sciences (BES), under Award No. DE-SC0019769. J.P. thanks the Sloan Foundation for support through Grant No. FG-2017-9392.

\section{APPENDIX A: EXPERIMENTAL METHODS}

The experiments are performed in microfluidic mazes designed using the KLAYOUT software. The mazes are networks of 5- $\mu \mathrm{m}$-wide paths, connected to side channels at the entrance and exit [Fig. 2(a)]. The side channels are $100 \mu \mathrm{m}$ wide and connected to reservoirs that supplied them with solutions. The height of the channels is 5-6 $\mu \mathrm{m}$. The molds are fabricated via a standard soft lithography process at the Nano3 Microfluidic Medical Device Facility at the University of California San Diego, where a Minitech 4-axis CNC micromilling machine (step size resolution $0.1 \mu \mathrm{m}$ ) prints the designs on 4-inch silicon wafers with SU-8 photoresist. The molds are cast with polydimethylsiloxane (PDMS, Sylgard 184, Dow Corning) and baked for two hours at 80-100 C.

An aqueous solution of $\mathrm{LiCl}$ with varying concentrations $(0-6 \mathrm{mM})$ is supplied to the reservoirs that set the boundary conditions at the entrance and exit for the maze. The channel at the entrance of the maze is also supplied with fluorescent polystyrene colloids (Invitrogen Fluo-Spheres ${ }^{\mathrm{TM}}$ carboxylate-modified microspheres), $1 \mu \mathrm{m}$ in diameter. We also include 3-(trimethoxysilyl) propyl methacrylate (TPM) spheres coated with a polymer brush (pluronic) that prohibits phoresis in our salt concentration, providing the means to monitor the flow. Finally, $0.6 \mathrm{mM}$ of tetramethylammonium hydroxide (TMAH) is used in all solutions to stabilize the colloids. The channels are filled with solution via a syringe pump (KD Scientific, model no. 180). A pressure controller (Fluigent MFCS-4C, 25 mbar) balances the pressure in the side channels to ensure the absence of any flows in the maze. This is further verified by the pluronic-coated TPM spheres, which diffuse randomly (in the event of a flow, they will be advected by the flow).

The system is observed via a 40X objective on a Nikon Eclipse Ti microscope, with an EO-1312C camera to record the experiments. The fluorescent colloids are excited at $470 \mathrm{~nm}$ and videos are recorded at $20 \mathrm{fps}$, making it easy to track the trajectories of the particles using MATLAB.

\section{APPENDIX B: STOCHASTIC MODEL}

With the given geometry and boundary conditions, the salt concentration is solved with COMSOL Multiphysics 5, which is also used for solving the mean first passage time equations. Once the concentration field $c$ is determined, the particle density associated with the phoretic velocity, $\mathbf{v}=D_{D P} f(c) \nabla c$, can be determined as $\rho(c)=C_{1} \int_{c}^{1} \exp \left[-\left(D_{D P} / D\right) \int_{c}^{u} f(s) d s\right] d u+$ $C_{2} \exp \left[-\left(D_{D P} / D\right) \int_{c}^{1} f(s) d s\right]$, with the constants $C_{1}$ and $C_{2}$ determined by the entrance and exit particle density.

The stochastic differential equation in Fig. 2 is integrated with the parameters $D=0.4$ and $D_{D P}=400 \mu \mathrm{m}^{2} / \mathrm{s}$, comparable to the experimental values. The network in simulations has the same geometry as in the experiments, except that all the dead ends have been removed to ensure computational efficiency. All the collisions between the particle and the network are fully elastic, which corresponds to the condition of a vanishing probability flux. Typically, one million particles are simulated to ensure the convergence of stochastic quantities.

The junction in Fig. 3 has the channel width $d=5 \mu \mathrm{m}$, with the ratio of salt concentration gradient to the left and right $2: 1$. Collisions are also fully elastic. The typical ratio of $D_{D P} / D=$ 1000 is fixed in all simulations. Each data point is sampled through 1000 stochastic simulations. 
The chemotaxis model evolves a three-state function $\left(\rho_{q}, \rho_{a}, \rho_{i}\right)$, where the subscription labels the quiescent, activated, and inhibited states of the surface detection [37]. They are defined on the surface of the phoretic particle and evolve according to the differential equations $\partial_{t} \rho_{q}=-\alpha c \rho_{q}+$ $\beta_{f} \rho_{i}, \partial_{t} \rho_{a}=\alpha c \rho_{q}-\delta \rho_{a}$ and $\partial_{t} \rho_{i}=-\beta_{f} \rho_{i}+\delta \rho_{a}$. In our example, we choose $\mathbf{v} \sim \oint \rho_{a} \nabla c$ so that the phoretic velocity is averaged on the surface of the particle, and the active area has more weight in the averaging. The parameters in the simulation are $\alpha=5 \mathrm{~s}^{-1}, \beta_{f}=0.01 \mathrm{~s}^{-1}$, and $\delta=0.1 \mathrm{~s}^{-1}$, similar to previous studies [37].

The run-and-tumble model measures the concentration profile $c(s)$ along the particle trajectory, where $s$ is the arc length measured from the particle center. The run-and-tumble particles move straight with a random amount of time that is a Poisson process with rate function $\omega$, after which the particles tumble to a random direction and resume running towards the new direction. We used the model in [40], where the rate function is determined by a convolution between the measured concentration profile $c(s)$ and a memory kernel $K$, such that $\omega=\tau^{-1}\left[1-\int^{t} K(t-s) c(s) d s\right]$ and $\tau^{-1}=1 \mathrm{~s}^{-1}$ is an intrinsic tumble rate. This mechanism detects the concentration gradient through the distribution of $c(s)$, which results in low tumbling rate when the particle moves along $\nabla c$. We performed simulations for $\tau^{-1}=0.1-2 \mathrm{~s}^{-1}$ without significant change of selectivity at the junction (see the Supplemental Material [41]). For particles with size, we use the average concentration on the particle surface as the signal $c(s)$, which, due to MVP, is simply the value of salt concentration measured at the particle center. We further tested the selectivity of run-and-tumble models at different parameters $\tau$ and $\beta$ and show that the selectivity at the junction is not sensitive to the choice of parameters (see the Supplemental Material [41]).

The run-and-whirl particle runs straight for a random period of time that is Poisson distributed with rate $\beta$, and it makes either a left- or a right-hand turn to complete a full circle of whirling with radius $r_{t}$. With the value of $\nabla c$ known on this circle, its value at the center can be determined as the average (via the MVP property). Upon completing the loop, the particle continues to run straight in the same direction as $\nabla c$. Upon finishing the loop, the particle averages $\nabla c$ along the circular path, allowing one to determine the harmonic function through boundary integrals.

[1] Howard C. Berg, E. coli in Motion (Springer, New York, 2008).

[2] H. Levine and W.-J. Rappel, The physics of eukaryotic chemotaxis, Phys. Today 66, 24 (2013).

[3] D. B. Dusenbery, Minimum size limit for useful locomotion by free-swimming microbes, Proc. Natl. Acad. Sci. USA 94, 10949 (1997).

[4] J. E. Segall, S. M. Block, and H. C. Berg, Temporal comparisons in bacterial chemotaxis, Proc. Natl. Acad. Sci. USA 83, 8987 (1986).

[5] I. Lagzi, S. Soh, P. J. Wesson, K. P. Browne, and B. A. Grzybowski, Maze solving by chemotactic droplets, J. Am. Chem. Soc. 132, 1198 (2010).

[6] C. Jin, C. Krüger, and C. C. Maass, Chemotaxis and autochemotaxis of self-propelling droplet swimmers, Proc. Natl. Acad. Sci. 114, 5089 (2017).

[7] H. Yamada, A. Toth, and T. Nakagaki, Intelligence: Maze-solving by an amoeboid organism, Nature (London) 407, 470 (2000).

[8] O. Steinbock, Á. Tóth, and K. Showalter, Navigating complex labyrinths: Optimal paths from chemical waves, Science 267, 868 (1995).

[9] W. Engl, M. Roche, A. Colin, P. Panizza, and A. Ajdari, Droplet Traffic at a Simple Junction at Low Capillary Numbers, Phys. Rev. Lett. 95, 208304 (2005).

[10] M. J. Fuerstman, P. Deschatelets, R. Kane, A. Schwartz, P. J. A. Kenis, J. M. Deutch, and G. M. Whitesides, Solving mazes using microfluidic networks, Langmuir 19, 4714 (2003).

[11] E. Um, J. M. Oh, J. Park, T. Song, T.-E. Kim, Y. Choi, C. Shin, D. Kolygina, J.-H. Jeon, B. A. Grzybowski, and Y.-K. Cho, Immature dendritic cells navigate microscopic mazes to find tumor cells, Lab Chip 19, 1665 (2019). 
[12] E. J. Marsden, C. Valeriani, I. Sullivan, M. E. Cates, and D. Marenduzzo, Chemotactic clusters in confined run-and-tumble bacteria: A numerical investigation, Soft Matter 10, 157 (2014).

[13] S. Park, P. M. Wolanin, E. A. Yuzbashyan, H. Lin, N. C. Darnton, J. B. Stock, P. Silberzan, and R. Austin, Influence of topology on bacterial social interaction, Proc. Natl. Acad. Sci. USA 100, 13910 (2003).

[14] G. B. Cunningham, V. Strauss, and P. G. Ryan, African penguins (Spheniscus demersus) can detect dimethyl sulphide, a prey-related odour, J. Expt. Biol. 211, 3123 (2008).

[15] F. Bonadonna, Evidence that blue petrel, Halobaena caerulea, fledglings can detect and orient to dimethyl sulfide, J. Expt. Biol. 209, 2165 (2006).

[16] B. DeLacyCostello and A. I. Adamatzky, Assessing the chemotaxis behavior of Physarum polycephalum to a range of simple volatile organic chemicals, Commun. Integrat. Biol. 6, e25030 (2013).

[17] J. Qin and A. R. Wheeler, Maze exploration and learning in C. elegans, Lab Chip 7, 186 (2007).

[18] M. M. Salek, F. Carrara, V. Fernandez, J. S. Guasto, and R. Stocker, Bacterial chemotaxis in a microfluidic T-maze reveals strong phenotypic heterogeneity in chemotactic sensitivity, Nat. Commun. 10, 1877 (2019).

[19] S. Palagi and P. Fischer, Bioinspired microrobots, Nat. Rev. Mater. 3, 113 (2018).

[20] P. Erkoc, I. C. Yasa, H. Ceylan, O. Yasa, Y. Alapan, and M. Sitti, Mobile microrobots for active therapeutic delivery, Adv. Therapeut. 2, 1800064 (2018).

[21] L. Tweedy, P. A. Thomason, P. I. Paschke, K. Martin, L. M. Machesky, M. Zagnoni, and R. H. Insall, Seeing around corners: Cells solve mazes and respond at a distance using attractant breakdown, Science 369, eaay9792 (2020).

[22] J. L. Anderson, Colloid transport by interfacial forces, Annu. Rev. Fluid Mech. 21, 61 (1989).

[23] B. Abecassis, C. Cottin-Bizonne, C. Ybert, A. Ajdari, and L. Bocquet, Boosting migration of large particles by solute contrasts, Nat. Mater. 7, 785 (2008).

[24] J. Palacci, B. Abecassis, C. Cottin-Bizonne, C. Ybert, and L. Bocquet, Colloidal Motility and Pattern Formation under Rectified Diffusiophoresis, Phys. Rev. Lett. 104, 138302 (2010).

[25] S. Battat, J. T. Ault, S. Shin, S. Khodaparast, and H. A. Stone, Particle entrainment in dead-end pores by diffusiophoresis, Soft Matter 15, 3879 (2019).

[26] S. Shin, O. Shardt, P. B. Warren, and H. A. Stone, Membraneless water filtration using CO2, Nat. Commun. 8, 1 (2017).

[27] M. Youssef, A. Morin, A. Aubret, S. Sacanna, and J. Palacci, Rapid characterization of neutral polymer brush with a conventional zetameter and a variable pinch of salt, Soft Matter 16, 4274 (2020).

[28] J. C. Crocker and D. G. Grier, Methods of digital video microscopy for colloidal studies, J. Colloid Interface Sci. 179, 298 (1996).

[29] S. Shin, P. B. Warren, and H. A. Stone, Cleaning by Surfactant Gradients: Particulate Removal from Porous Materials and the Significance of Rinsing in Laundry Detergency, Phys. Rev. Appl. 9, 034012 (2018).

[30] J. Palacci, C. Cottin-Bizonne, C. Ybert, and L. Bocquet, Osmotic traps for colloids and macromolecules based on logarithmic sensing in salt taxis, Soft Matter 8, 980 (2012).

[31] S. Shin, E. Um, B. Sabass, J. T. Ault, M. Rahimi, P. B. Warren, and H. A. Stone, Size-dependent control of colloid transport via solute gradients in dead-end channels, Proc. Natl. Acad. Sci. USA 113, 257 (2016).

[32] J.-L. Barrat and J. Pierre Hansen, Basic Concepts for Simple and Complex Liquids (Cambridge University Press, Cambridge, 2003).

[33] S. Redner, A Guide to First-Passage Processes (Cambridge University Press, Cambridge, 2001).

[34] D. C. Prieve and R. Roman, Diffusiophoresis of a rigid sphere through a viscous electrolyte solution, J. Chem. Soc., Faraday Trans. 2 83, 1287 (1987).

[35] H. J. Keh and S. B. Chen, Electrophoresis of a colloidal sphere parallel to a dielectric plane, J. Fluid Mech. 194, 377 (2006).

[36] H.-J. Keh and J. L. Anderson, Boundary effects on electrophoretic motion of colloidal spheres, J. Fluid Mech. 153, 417 (1985).

[37] W. J. Rappel, P. J. Thomas, H. Levine, and W. F. Loomis, Establishing direction during chemotaxis in eukaryotic cells, Biophys. J. 83, 1361 (2002). 
[38] D. Takagi, A. B. Braunschweig, J. Zhang, and M. J. Shelley, Dispersion of Self-Propelled Rods Undergoing Fluctuation-Driven Flips, Phys. Rev. Lett. 110, 038301 (2013).

[39] B. ten Hagen, F. Kümmel, R. Wittkowski, D. Takagi, H. Löwen, and C. Bechinger, Gravitaxis of asymmetric self-propelled colloidal particles, Nat. Commun. 5, 4829 (2014).

[40] A. Celani and M. Vergassola, Bacterial strategies for chemotaxis response, Proc. Nat. Acad. Sci. USA 107, 1391 (2010).

[41] See Supplemental Material at http://link.aps.org/supplemental/10.1103/PhysRevFluids.5.104202 for supplemental movies and information as described in the main text. 